\title{
Inventário dos Brachyura de substratos consolidados naturais do mesolitoral da Praia do Coqueiro, Luís Correia - Piauí
}

\author{
Tarcísio Barbosa Lima Júnior ${ }^{1 *}$ \\ Mona Indianara da Costa Aragão ${ }^{1,2}$ \\ José Roberto de Souza de Almeida Leite ${ }^{1}$ \\ Tito Monteiro da Cruz Lotufo ${ }^{3}$ \\ Gustavo Augusto Schmidt de Melo ${ }^{4}$ \\ Projeto Biodiversidade do Delta Conservação e Pesquisa - PROBID \\ Grupo de Biodiversidade e Biotecnologia, Universidade Federal do Piauí \\ Campus Ministro Reis Velloso, CEP 64202-020, Parnaíba - PI, Brasil \\ ${ }^{2}$ Campus Professor Alexandre Alves de Oliveira \\ Universidade Estadual do Piauí, Parnaíba - PI, Brasil \\ ${ }^{3}$ Laboratório de Ecologia Animal, Instituto de Ciências do Mar \\ Universidade Federal do Ceará, Fortaleza - CE, Brasil \\ ${ }^{4}$ Museu de Zoologia da Universidade de São Paulo, São Paulo - SP, Brasil \\ *Autor para correspondência \\ tjpesca@gmail.com
}

Submetido em 29/08/2009

Aceito para publicação em 22/02/2010

\section{Resumo}

A composição dos Brachyura ao longo de substratos consolidados naturais na Praia do Coqueiro no litoral de Luís Correia (Piauí) é apresentada. Neste estudo foram registradas 20 espécies de Brachyura, das quais oito nunca haviam sido registradas para o estado: Epialtus bituberculatus H. Milne Edwards, 1834; Acantholobulus schmitti (Rathbun), 1930; Pachygrapsus transversus (Gibbes, 1850); Panopeus austrobesus Williams, 1983; Panopeus occidentalis Saussure, 1857; Pilumnus dasypodus Kingsley, 1879; Pilumnus spinosissimus Rathbun, 1898 e Planes cyaneus Dana, 1851; sendo registrada também a espécie Charybdis hellerii (Milne Edwards, 1867), de origem Indo-Pacífica. A família com maior representatividade foi Xanthidae, com nove espécies de caranguejos, seguida por Grapsidae (3), Portunidae (4) e Majidae com duas espécies, e Calappidae e Ocypodidae com uma espécie identificada.

Unitermos: Brachyura, inventário, mesolitoral, Piauí, substratos consolidados

\section{Abstract}

Inventory of Brachyura from natural mesolittoral hard substrata of Coqueiro Beach, Luís Correia, Piauí. The composition of Brachyura inhabiting natural hard substrata at Coqueiro Beach, on the coast of Luís Correia (Piauí), is presented. A total of 20 species of Brachyura were recorded, eight of which were not previously registered for the state: Epialtus bituberculatus H. Milne Edwards, 1834; Acantholobulus schmitti (Rathbun, 1930); Pachygrapsus transversus (Gibbes, 1850); Panopeus austrobesus Williams, 1983; Panopeus occidentalis Saussure, 1857; Pilumnus dasypodus Kingsley, 1879; Pilumnus spinosissimus Rathbun, 1898; and Planes cyaneus 
Dana, 1851. The Indo-Pacific species Charybdis hellerii (Milne Edwards, 1867) was also identified. The most diverse family was Xanthidae with nine species of crabs, followed by Grapsidae (3), Portunidae (4), Majidae with two species, and Calappidae and Ocypodidae with a single species each.

Key words: Brachyura, composition, hard substrata, mesolittoral, Piauí

\section{Introdução}

Ao longo de sua pequena extensão litorânea, cerca de $66 \mathrm{~km}$, o estado do Piauí apresenta diversas praias com costões extensos, como é o caso da praia do Coqueiro, localizada no litoral de Luís Correia. Esses afloramentos possuem uma biota expressiva, com grande abundância de macroalgas e animais marinhos. Por se encontrarem em uma região tropical, essas áreas têm potencialmente uma grande diversidade biológica que permanece em grande parte ignorada. Estas áreas têm sofrido forte impacto antrópico, por estarem acessíveis à população litorânea e sujeitas a problemas oriundos das alterações da hidrodinâmica costeira.

Investigações sobre a composição da fauna de ambientes bentônicos têm sido propostas como instrumentos essenciais em programas de biomonitoramento para a avaliação da qualidade ambiental, sendo capazes de orientar medidas mitigadoras para os efeitos negativos causados por atividades antrópicas e de fornecer subsídios práticos para o gerenciamento desses ambientes (Warwick e Radford, 1989).

Dentre os diversos grupos de animais que habitam estes locais, destacam-se os crustáceos decápodos braquiúros por sua contribuição fundamental para a complexidade e funcionamento dos ecossistemas tropicais (Hendrickx, 1995). Isto se dá tanto pela sua participação na reciclagem de nutrientes e matéria orgânica depositados, quanto pela sua utilização como itens alimentares pelo homem ou por peixes demersais de importância econômica (Caddy, 1989).

A literatura sobre os decápodos da costa brasileira trata com frequência de áreas geográficas restritas. Apesar disso, tais trabalhos são importantes para o entendimento dos vários aspectos das comunidades bentônicas, além de fornecer um conhecimento básico sobre a biologia destes organismos (Braga et al., 2005).
Ao longo dos últimos anos, muitos pesquisadores brasileiros na área de carcinologia estudaram os braquiúros abordando os mais variados assuntos. Dentre eles, como importantes levantamentos faunísticos podemos citar: Moreira (1903), Coelho e Ramos (1972), Melo et al., (1989), Cobo et al., (1993); e citando novas ocorrências: Oliveira (1944), Gouvêa (1971), Melo (1983), Góes et al. (1998), Bezerra et al., (2005), Oliveira e Oshiro (2006), Góes e Fernandes-Góes (2007). Outros trabalhos abordaram especificamente a fauna de decápodos dos estuários do nordeste brasileiro, entre os quais: Rathbun (1900), Coelho (1965/66), Ramos-Porto et al. (1978), Coelho e Ramos-Porto (1980), Coelho et al. (1986), Coelho e Coelho-Santos (1990), CoelhoSantos e Coelho (2001), Coelho et al. (2002), Ferreira e Sankarankutty (2002) e Calado e Sousa (2003).

Alguns estados no nordeste do Brasil apresentam a fauna de crustáceos decápodos estuarina bem conhecida, com destaque para Pernambuco e Alagoas. Já no Maranhão e Piauí, o conhecimento sobre a composição da carcinofauna é incipiente (Almeida et al., 2006).

De maneira a reduzir a lacuna que existe sobre a fauna de Brachyura no Piauí, foi iniciado em 2007 o subprojeto "Caracterização carcinológica da costa do Estado do Piauí", como parte do Projeto Biodiversidade do Delta - PROBID da Universidade Federal do Piauí (UFPI) em Parnaíba. O presente trabalho teve como objetivo o estudo da composição específica, em ambientes consolidados naturais, da fauna de Brachyura no litoral do município de Luís Correia no estado do Piauí, nordeste do Brasil.

\section{Material e Métodos}

Para o levantamento foram realizadas coletas entre os meses de agosto e dezembro de 2007, na praia do Coqueiro, Luís Correia (Piauí), localizada entre as coordenadas $02^{\circ} 51,1^{\prime} \mathrm{S}$ e $041^{\circ} 38,7^{\prime} \mathrm{W}$ (Figura 1). As amostragens 


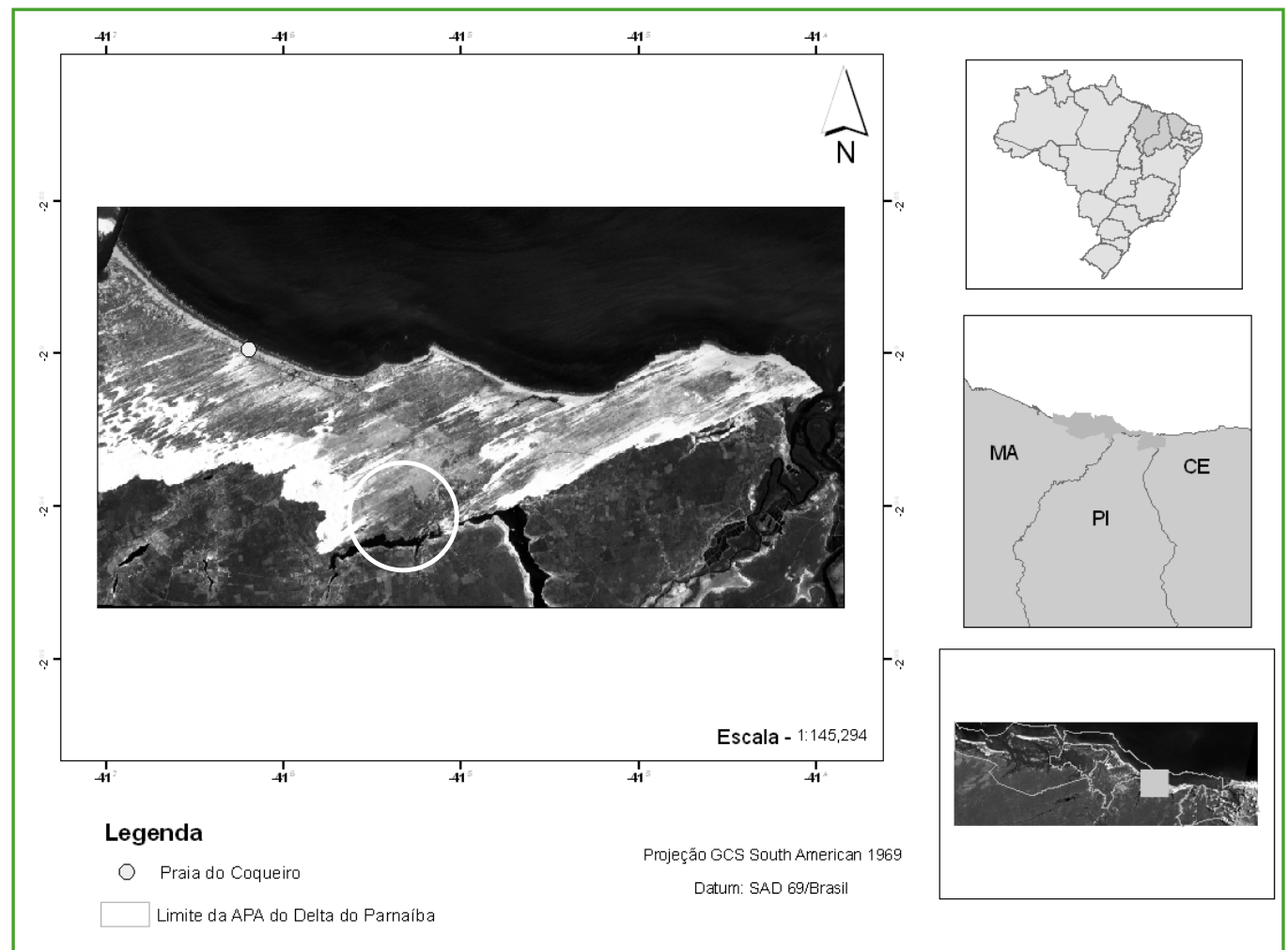

FIGURA 1: Mapas indicativos dos locais de coletas (ć́rculo indica o local de coleta em substratos consolidados) referentes à zona costeira da Praia do Coqueiro no litoral de Luís Correia, Piauí, Brasil.

foram qualitativas, sem padronização de esforço, foram visitados os substratos mais significativos em termos de extensão. Os Brachyura foram coletados sob a licença de número 12529-1/SISBIO. Os animais foram capturados manualmente durante as baixamares de sizígia, sendo em seguida congelados em refrigerador a $-8^{\circ} \mathrm{C}$, de maneira a se preservar a coloração. Após o congelamento foram fixados em formol a $4 \%$ e posteriormente conservados em álcool etílico $70 \%$. Os exemplares foram identificados com o auxílio de um microscópio estereoscópico e chaves dicotômicas específicas (Williams, 1984; Melo, 1996). Os animais capturados foram depositados na coleção zoológica da UFPI.

\section{Resultados e Discussão}

Neste levantamento foram coletadas 20 espécies de Brachyura, a saber:

Família Calappidae De Haan, 1833

Calappa sulcata Rathbun, 1898
Família Grapsidae MacLeay, 1838

Pachygrapsus gracilis (Saussure, 1858)

Pachygrapsus transversus (Gibbes, 1850)

Planes cyaneus Dana, 1851

Família Majidae Samouelle, 1819

Epialtus bituberculatus H. Milne Edwards, 1834

Microphrys bicornutus (Latreille, 1825)

Família Ocypodidae Rafinesque, 1815

Uca leptodactyla Rathbun, 1898

Família Portunidae Rafinesque, 1815

Callinectes danae Smith, 1869

Callinectes exasperatus (Gerstaecker, 1856)

Callinectes larvatus Ordway, 1863

Charybdis hellerii (Milne Edwards, 1867)

Família Xanthidae MacLeay, 1838

Eriphia gonagra (Fabricius, 1781)

Acantholobulus schmitti (Rathbun, 1930)

Menippe nodifrons Stimpson, 1859 
Panopeus americanus Saussure, 1857

Panopeus austrobesus Williams, 1983

Panopeus occidentalis Saussure, 1857

Pilumnus dasypodus Kingsley, 1879

Pilumnus reticulatus Stimpson, 1860

Pilumnus spinosissimus Rathbun, 1898

A família com maior representatividade foi Xanthidae, com nove espécies de caranguejos, seguida por Grapsidae e Portunidae, com quatro espécies, Majidae com duas espécies, e Calappidae e Ocypodidae com uma espécie cada.

O estudo não apresenta nenhuma conotação quantitativa, levando em consideração que o local de estudo trata-se de uma Área de Proteção Ambiental Federal, mas é válido ressaltar que no desenvolvimento das coletas foi observado que as espécies Menippe nodifrons, Pachygrapsus transversus e Panopeus americanus se destacaram por apresentar visivelmente uma maior abundância.

A espécie Charybdis hellerii (Milne Edwards, 1867) é aqui registrada novamente para a costa do Estado do Piauí, tendo sido registrada pela primeira vez em 2007 (Lima Júnior et al., 2009), onde foi identificada em um sistema estuarino da Praia de Macapá.

Das oito espécies de decapódos marinhos reconhecidas como exóticas e relacionadas por Tavares e Mendonça Jr. (1996), apenas duas espécies de Brachyura, Charybdis hellerii (A. Milne Edwards, 1867) e Pyromaia tuberculata (Lockington, 1877), cujas populações são bastante numerosas na costa brasileira, tiveram relativo êxito na sua introdução (Melo e Crivelaro, 2002). O portunídeo Charybdis hellerii (A. Milne Edwards, 1867) é um dos diversos casos graves de introdução que está prejudicando a pesca de espécies nativas na Bahia. Além disso, existe o risco de que ele se torne um competidor das espécies comercialmente importantes e desencadeie alterações na estrutura e composição taxonômica nas populações (Tavares e Mendonça Jr., 2004). Vale ressaltar que no local de estudo foram identificadas outras espécies de portunídeos, configurando a importância de levantamentos regulares na região com intuito de se analisar o impacto desta espécie no ambiente e a relação com as espécies nativas.
Em um levantamento realizado na costa do Ceará (SEMACE/LABOMAR, 2005), a localidade com maior diversidade de braquiúros foi na praia do Meireles, em Fortaleza, com um total de 15 espécies. Deste total, apenas cinco espécies são comuns a este estudo, o que aponta para uma diferença importante em termos da composição faunística, mesmo tratando-se de regiões próximas geograficamente.

Até o momento algumas espécies da fauna de braquiúros encontradas corresponderam aos que já se conhecia para o litoral piauiense. Por outro lado, nove espécies encontradas não possuíam registro para o estado do Piauí, sendo assim, o novo registro foi incluído na distribuição geográfica das mesmas, isso poderá ser observado com destaque ao longo do texto.

De acordo com Góes et al. (1998), o fato de alguns caranguejos apresentarem uma distribuição em áreas de difícil acesso, aliado à escassez de trabalhos sobre o conhecimento da composição faunística, promove registros tardios da biodiversidade.

As espécies registradas pela primeira vez no estado do Piauí são:

Epialtus bituberculatus H. Milne Edwards, 1834. Distribuição geográfica: Atlântico ocidental - Flórida, Golfo do México, Antilhas, Colômbia, Venezuela e Brasil (do Piauí até São Paulo).

Acantholobulus schmitti (Rathbun, 1930). Distribuição geográfica: Atlântico ocidental - Brasil (do Piauí até Santa Catarina) e Uruguai (Melo, 1996).

Panopeus austrobesus Williams, 1983. Distribuição geográfica: Atlântico ocidental - Brasil (do Piauí e do Rio de Janeiro ao Rio Grande do Sul) (Melo, 1996).

Panopeus occidentalis Saussure, 1857. Distribuição geográfica: Atlântico ocidental - Carolina do norte até a Flórida, Golfo do México, América Central, Antilhas, norte da América do Sul, Guianas e Brasil (do Piauí até Santa Catarina) (Melo, 1996).

Pachygrapsus transversus (Gibbes, 1850). Distribuição geográfica: Atlântico ocidental - Bermuda, da Carolina do Norte até a Flórida, Golfo do México, Antilhas, norte da América do Sul, Brasil (Trindade e do Piauí até o Rio Grande do Sul) e Uruguai. Atlântico 
oriental - Ilhas de Cabo Verde até Angola. Mar Mediterrâneo. Pacífico oriental - Califórnia até o Peru (Melo, 1996).

Pilumnus dasypodus Kingsley, 1879. Distribuição geográfica: Atlântico ocidental - Carolina do Norte e do Sul, Flórida, Golfo do México, Antilhas, norte da América do Sul e Brasil (do Piauí e da Paraíba até Santa Catarina) (Melo, 1996).

Pilumnus spinosissimus Rathbun, 1898. Distribuição geográfica: Atlântico ocidental - Flórida, Golfo do México, Antilhas e Brasil (do Piauí e do Rio Grande do Norte até Santa Catarina) (Melo, 1996).

Planes cyaneus Dana, 1851. Distribuição geográfica: Atlântico ocidental - Brasil (do Piauí e do Rio Grande do Norte ao Rio Grande do Sul), Uruguai e Argentina. Atlântico oriental - África Tropical e ilha de Santa Helena. Indo-Pacífico e Pacífico oriental (Williams, 1984; Melo, 1996).

Segundo Melo (1996), P. cyaneus é uma espécie que ocorre em mar aberto presa a objetos flutuantes e algas, mas ocasionalmente pode ser encontrada em restos de navios que dão à praia, resultando numa distribuição dependente das correntes marítimas.

Pilumnus spinosissimus possui como habitat natural substratos de areia, de rochas e principalmente de corais, podendo ser encontrada de 5 a $20 \mathrm{~m}$ de profundidade. No local de estudo, esta espécie foi coletada na franja do infralitoral.

As espécies E. bituberculatus, A. schmitti, $P$. occidentalis, e P. transversus possuem distribuição citada para o estado do Ceará, indicando um registro tardio para a costa do estado do Piauí por falta de trabalhos relacionados a levantamentos faunísticos e monitoramentos adequados.

Na região de estudo foi observado que as espécies Panopeus occidentalis, P. americanus e Menippe nodifrons são capturadas para consumo. Esta atividade, chamada de "mariscagem", segundo os pescadores locais, é praticada durante o ano todo por ocasião das marés de sizígia e é destinada à própria subsistência, sendo considerada um recurso pesqueiro sem valor comercial.
A riqueza de espécies desaparece rapidamente e, no contexto atual, as informações sobre a biodiversidade são indispensáveis para subsidiar políticas de conservação, principalmente considerando que a região está dentro dos limites da Área de proteção Ambiental Delta do Parnaíba (APA Delta).

Os futuros inventários de biodiversidade têm, necessariamente, de ser planejados em torno de procedimentos de estimativas de riqueza, pois as transformações drásticas nos ambientes e as altas taxas de extinção da biota irão requerer um grande volume de trabalho humano na área de sistemática e ecologia, a fim de que os diferentes grupos de organismos possam ser incorporados aos planos de conservação (Coddington et al., 1991; Colwell e Coddington, 1994).

Os dados aqui gerados poderão direcionar futuros trabalhos sobre a biologia e ecologia da fauna de Brachyura. Em futuros estudos, provavelmente novas coletas na costa nesse estado revelem outros registros, dado o desconhecimento em relação à fauna da região. Estas informações preenchem em parte a lacuna existente sobre a fauna de Brachyura no estado do Piauí e amplia setentrionalmente o conhecimento biogeográfico das espécies E. bituberculatus, Acantholobulus schmitti, Panopeus austrobesus, P. occidentalis, Pachygrapsus transversus, Pilumnus dasypodus, P. spinosissimus e Planes cyaneus para a costa deste estado, mais precisamente, para o litoral do município de Luís Correia.

\section{Agradecimentos}

Os autores agradecem ao Projeto Biodiversidade do Delta (PROBID), Universidade Federal do Piauí (CMRV/Parnaíba) e Programa Pesquisador UFPI/2007 pelo apoio.

\section{Referências}

Almeida, A. O.; Coelho, P. A.; Santos, J. T. A.; Ferraz, N. R. 2006. Crustáceos decápodos estuarinos de Ilhéus, Estado da Bahia, Brasil. Biota Neotropica, 6 (2).

Bezerra, L. E. A.; Almeida, A. O.; Coelho, P. A. 2005. Primeiro registro de Apiomithrax violaceus (A. Milne Edwards) e Hypoconcha arcuata Stimpson (Crustacea, Decapoda, Brachyura) 
para o litoral do Ceará, Brasil. Revista Brasileira de Zoologia, 22 (4): 919-922.

Braga, A. A.; Fransozo, A.; Bertini, G.; Fumis, P. B. 2005. Composição e abundância dos caranguejos (Decapoda, Brachyura) nas regiões de Ubatuba e Caraguatatuba, litoral norte paulista, Brasil. Biota Neotropica, 5 (2): 45-78.

Caddy, J. F. 1989. Recent developments in research and management for wild stocks of bivalves and gastropods. In: Caddy, J. F. (Ed.). Marine invertebrate fishery: Their assessment and management. John Wiley e Sons, London, UK, p.665-699.

Calado, T. C. S.; Sousa, E. C. 2003. Crustáceos do Complexo Estuarino-lagunar Mundaú/Manguaba Alagoas. Fapeal, Maceió, Brasil, p.116.

Cobo, V. J.; Fransozo, A.; Mantelatto, F. L. M.; Pinheiro, M. A. A.; Santos, S.; Góes, J. M. 1993. Composição dos braquiúros (Crustacea, Decapoda) no manguezal formado pelos rios Comprido e Escuro, Ubatuba, SP. Anais do III Simpósio de Ecossistema da Costa Brasileira, Serra Negra, Brasil, p.146-150.

Coddington, J. A.; Griswold, C. E.; Silva, D.; Peñarada, E.; Archer, S. F. 1991. Designing and testing sampling protocols to estimate biodiversity in tropical ecosystems. In: Dudley, E. C. (Ed.). The unity of Evolutionary Biology: Proceedings Fourth International Congress of Systematic and Evolutionary Biology, Dioscorides Press, Portland, USA, p.44-60.

Coelho, P. A. 1965/66. Os crustáceos decápodos de alguns manguezais de Pernambuco. Trabalhos Oceanográficos da UFPE, 7/8: 71-90.

Coelho, P. A.; Coelho-Santos, M. A. 1990. Crustáceos decápodos e estomatópodos do rio Paripe, Itamaracá - PE. Anais da Sociedade Nordestina de Zoologia, 3: 43-61.

Coelho, P. A.; Ramos, M. A. 1972. A constituição e a distribuição da fauna de decápodos do litoral leste da América do Sul entre as latitudes $5^{\circ} \mathrm{N}$ e $39^{\circ} \mathrm{S}$. Trabalhos Oceanográficos da UFPE, 13: 133-236.

Coelho, P. A.; Ramos-Porto, M. 1980. Crustáceos decápodos da costa do Maranhão, Brasil. Boletim do Instituto de Oceanografia, 29 (2): 135-138.

Coelho, P. A.; Ramos-Porto, M.; Calado, T. C. S. 1986. Litoral do Rio Grande do Norte: Decapoda. Caderno Ômega da Universidade Federal Rural de Pernambuco - Série Ciências Aquáticas, 2: 79-105.

Coelho, P. A.; Santos, M. A. C.; Torres, M. F. A.; Monteiro, B. R.; Almeida, V. A. K. 2002. Reino Animalia: Filo (ou Subfilo) Crustacea no Estado de Pernambuco. In: Tabarelli, M. \& Silva, J. M. C. (Eds). Diagnóstico da biodiversidade de Pernambuco. v.2. Massangana, Recife, Brasil, p.429-482.

Coelho-Santos, M. A.; Coelho, P. A. 2001. Crustacea Decapoda of the Paripe river estuary, Pernambuco, Brazil. Hydrobiologia, 449 (1-3): 77-79.

Colwell, R. K.; Coddington, J. A. 1994. Estimating the extent of terrestrial biodiversity through extrapolation. Philosophical Transactions of the Royal Society of London B, 345: 101-118.

Ferreira, A. C.; Sankarankutty, C. 2002. Estuarine carcinofauna (Decapoda) of Rio Grande do Norte, Brazil. Nauplius, 10 (2): 121129.

Góes, J. M.; Fernandes-Góes, L. C. 2007. First occurrence of the crab Calappa nitida Holthuis, 1958 (Brachyura, Calappidae,
Calappinae) on the coast of the state of Piauí, Brazil. Biotemas, 20 (1): 123-125.

Góes, J. M.; Cobo, V. J.; Fransozo, A.; Mantelatto, F. L. M. 1998. Novas Ocorrências de Caranguejos Marinhos (Crustacea, decapoda Brachyura) para o Litoral de São Paulo. v.3. IV Simpósio de Ecossistemas Brasileiros, Águas de Lindóia, Brasil, p. 426-430.

Gouvêa, E. P. 1971. Ocorrência de Cyrtoplax spinidentata (Crustacea: Decapoda; Goneplacidae) no litoral brasileiro. Ciência e Cultura, 23 (Suplemento): 391.

Hendrickx, M. E. 1995. Checklist of brachyuran crabs (Crustacea: Decapoda) from the eastern tropical Pacific. Bulletin de l'Institut Royal des Sciences Naturelles de Belgique - Biologie, 65: 125-150.

Lima Júnior, T. B.; Aragão, M. I. C.; Silva, J. P.; Melo, G. A. S.; Leite, J. R. S. A. 2009. Occurrence of two indo-pacific species of Brachyura on the coast of Piauí, Brazil. Boletim do Laboratório de Hidrobiologia do LABOHIDRO, 21: 35-40.

Melo, G. A. S. 1983. A ocorrência no litoral brasileiro de um Portunidae (Crustacea: Decapoda: Brachyura) originário do IndoPacífico. Revista Brasileira de Zoologia, 1 (3): 159-167.

Melo, G. A. S. 1996. Manual de identificação dos Brachyura (caranguejos e siris) do litoral brasileiro. Plêiade Fapesp, São Paulo, Brasil. 604pp

Melo, G. A. S.; Veloso, V. G.; Oliveira, M. C. 1989. A fauna de Brachyura (Crustacea: Decapoda) do litoral do Estado do Paraná. Nerítica, 4 (1/2): 1-31.

Melo, G. A. S.; Crivelaro, T. B. 2002. Primeira ocorrência de Polybius navigator (Herbst) (Decapoda, Brachyura, Portunidae) no Atlântico Ocidental. Revista Brasileira de Zoologia, 19 (1): 233-238.

Moreira, C. 1903. Crustáceos da Ponta do Pharol, em São Francisco do Sul no Estado de Santa Catarina. Arquivo do Museu Nacional do Rio Janeiro, 12: 119-123.

Oliveira, L. P. H. 1944. Sobre a existência na baia do Rio de Janeiro de uma aranha originária do Oceano Pacífico: Libinia rostrata Bell, 1835 (Majidae: Decapoda). Memórias do Instituto Oswaldo Cruz, 40 (1): 87-90.

Oliveira, L. M. S.; Oshiro, L. M. Y. 2006. Observações sobre a ocorrência de Pilumnus floridanus Stimpson (Decapoda, Brachyura, Pilumnidae) na costa do Rio de Janeiro, Brasil. Revista Brasileira de Zoologia, 23 (4): 1256-1257.

Ramos-Porto, M.; Ferreira-Correira, M. M.; Sousa, N. R. 1978. Levantamento da fauna aquática da ilha de São Luís (Estado do Maranhão, Brasil). II. Crustacea. Boletim do Laboratório de Hidrobiologia, 2 (1): 77-88.

Rathbun, M. J. 1900. The decapod and stomatopod Crustacea $-1^{\circ}$ Results of the Branner-Agassiz Expedition to Brazil. Proceedings of the Washington Academy of Science, 2: 133-156.

Superintendência Estadual do Meio Ambiente - Laboratório de Ciências do Mar SEMACE/LABOMAR. 2005. Programa: Zoneamento Ecológico e Econômico (ZEE) da Zona Costeira do estado do Ceará - Levantamento da Macrofauna Bentônica de Ambientes Consolidados: Relatório Final. Governo do Estado do Ceará, Fortaleza, Brasil, p.111.

Tavares, M.; Mendonça Jr., J. B. 1996. Charybdis hellerii (A. Milne Edwards, 1867) (Brachyura: Portunidae), eighth nonindigenious marine decapod from Brazil. Crustacean Research, 25: 151-157. 
Tavares, M.; Mendonça Jr., J. B. 2004. Introdução de crustáceos decápodes exóticos no Brasil. In: Silva, J. S. V. \& Souza, R. C. C. L. de (Eds). Água de lastro e bioinvasão. Interciencia, Rio de Janeiro, Brasil, p.59-76.

Warwick, R. M.; Radford, P. J. 1989. Analysis of the flow network in estuarine benthic community. Coastal and Estuarine Studies. In: Wulff, F.; Field, J. G. \& Mann, K. H. (Eds). Network analysis in marine ecology-Methods and applications. Springer, Heidelberg, Germany, p.119-160.

Williams, A. B. 1984. Shrimps, lobsters, and crabs of the Atlantic Coast of the Eastern United States, Maine to Florida. Smithsonian Institution Press, Washington, USA, 550pp. 